\title{
The persistence of intracellular Staphylococcus aureus in the sinuses: a longitudinal study*
}

\author{
Judy Ou, Ahmed Bassiouni, Amanda Drilling, Alkis J. Psaltis, Sarah Vreugde, Rhinology 55:305-311, 2017 \\ Peter-John Wormald \\ https://doi.org/10.4193/Rhino16.218 \\ Department of Surgery-Otorhinolaryngology Head and Neck Surgery, The University of Adelaide, South Australia, Australia \\ *Received for publication: \\ July 15, 2016 \\ Accepted: May 7, 2017
}

\begin{abstract}
Background: Staphylococcus aureus (S. aureus) can reside within the sinonasal mucosa in chronic rhinosinusitis patients and causes recurrent infections. Within the host cell, S. aureus can evade host immune detection enabling its own survival. We hypothesise that $S$. aureus can persist within the sinonasal epithelium for a prolonged period without immune activation.

Methodology: Patients with chronic rhinosinusitis with nasal polyps (CRSwNP) undergoing two sinus surgeries were included. Immunohistochemistry and Haematoxylin and Eosin stains were used to determine intracellular S. aureus (ICSA) status and inflammatory cell count, respectively. One-way ANOVA and paired t-tests were performed for comparison between ICSA subgroups and within each subgroup, respectively.
\end{abstract}

Results: Histopathological specimens from 34 patients (68 procedures) were included. ICSA positivity (ICSA ${ }^{+}$) was seen in 43 specimens (63.2\%) from 26 (76\%) patients. 18 (52.9\%) of those were ICSA+ in both operations while $8(23.5 \%)$ patients were ICSA in only one of the operations. 8 (23.5\%) patients were ICSA negative in both operations. There was no difference in the number of eosinophils, lymphocyte and neutrophils between ICSA subgroups.

Conclusions: This study demonstrated that $S$. aureus is found intracellularly within CRSWNP tissue at multiple time points without an increase in the number of eosinophils, lymphocytes and neutrophils. This finding supports our hypothesis that ICSA is able to escape from host detection and resides within the sinonasal mucosa despite intense treatment.

Key words: chronic rhinosinusitis, Staphylococcus aureus, transnasal endoscopic surgery, inflammation, fibrosis

\section{Introduction}

Intracellular Staphylococcus aureus (ICSA) has been demonstrated within the sinonasal epithelium in patients with chronic rhinosinusitis (CRS) as well as in healthy individuals ${ }^{(1-5)}$. It is known that upon entrance into the cells, Staphylococcus aureus (S. aureus) undergoes significant phenotypic changes by modifying its metabolism and down regulating the expression of virulence factors ${ }^{(6)}$. A study conducted by Wood et al. ${ }^{(4)}$ showed a lack of local immune response to intramucosal microcolonies in patients with CRS, supporting the concept that these adaptation mechanisms allow its survival within the cells and facilitate its evasion from host immune detection. Further compounding this problem is the fact that antibiotics commonly used to treat methicillin sensitive S. aureus infection in the setting of CRS, such as $\beta$-lactams, have low intracellular activity and therefore are ineffective in eliminating ICSA ${ }^{(7)}$. The combination of these factors allows ICSA to remain undetected as well as survive and persist within the cells.

In vivo persistence of ICSA in CRS patients was firstly demonstrated by Clement et al. ${ }^{(1)}$ and Plouin-Gaudon et al. ${ }^{(2)}$. Both groups showed that the same patient specific genotypes of $S$. aureus were repeatedly cultured in patients that had ICSA. These findings indicate that one specific $S$. aureus strain dominates the niche and can cause recurrent infections. Further support of this finding was a study that showed identical S. aureus genotypes 
in CRS patients isolated from their sinonasal cavities at multiple time points ${ }^{(8)}$. Together, these findings support the notion that ICSA might act as a reservoir causing recurrent infections and may play a role in CRS pathogenesis. The long-term consequence of ICSA in CRS remains unclear although ICSA has been found to be more prevalent in CRS patients with surface biofilm ${ }^{(3)}$ and in those requiring revision surgery suggesting a role in recalcitrant disease ${ }^{(5,9)}$.

It is unknown whether the presence of ICSA is affected by current standard medical management of CRS. This study was designed with an aim to investigate the persistence of ICSA in patients with recalcitrant CRS who had been treated with intense medical and surgical therapies. The second aim of this study was to gain further understanding of the longitudinal changes in histopathological features, inflammatory cell load and fibrosis in association with ICSA.

\section{Materials and methods}

Ethics approval for this study was granted by the Human Research Ethics Committee of The Queen Elizabeth Hospital (South Australia, Australia).

\section{Patient recruitment}

Patients diagnosed with CRS with nasal polyps (CRSwNP) according to the 2012 European Position Paper on Rhinosinusitis and Nasal Polyps ${ }^{(10)}$ diagnostic criteria and had undergone two endoscopic sinus surgeries (ESS) between 2003 and 2015 were recruited into the study. Histopathological specimens of polyps taken at each surgical procedure were examined in the study. Specimens from patients who had a history of immunosuppression or were less than 18 years of age were excluded from the study. Clinical data including pre- and post-operative management was obtained from patient medical records.

Histology staining - haematoxylin and eosin (H\&E) and immunohistochemistry (IHC)

Archived histology blocks of sinonasal mucosa from each ESS were retrieved. Two consecutive sections of $4 \mu \mathrm{m}$ thickness were cut from each block and placed on separate slides. Specimens underwent both routine H\&E staining to assess inflammatory cell counts and basement membrane thickness and immunohistochemistry $(\mathrm{IHC})$ staining to determine ICSA status.

IHC was performed using an automated system Leica BOND-III (Leica Biosystems, Germany). The autostainer was programmed as follows: 15 minutes heat fix at $60^{\circ} \mathrm{C}$ followed by rehydration by changes of decreasing concentrations of ethanol. Dual endogenous peroxidase block (Dako EnVision + Dual Link SystemHRP, K4065) for 10 minutes at room temperature followed by 20 minutes of $\mathrm{pH} 6$ citrate buffer incubation at $98^{\circ} \mathrm{C}$ to antigen re- trieve. Primary mouse anti S. aureus antibody (Millipore MAB930) was used at 1 in 100 concentration for 1 hour at room temperature. Unimmunised mouse IgM (Sigma M2521) and normal saline were used as negative controls. 3,3'-Diaminobenzidine (DAB) stain was developed using Dako EnVision kit (K4065) as per manufacturer's instructions.

\section{Image analysis}

All stained slides were scanned into digital files using a NanoZoomer 2.0-HT (Hamamatsu, Japan) to allow whole image analysis. The images were reviewed using NDP.view2 software (Hamamatsu, Japan).

\section{ICSA status}

To determine the presence of ICSA, two blinded independent investigators (JO \& AD) assessed all IHC stained slides. The slides were coded with specimen identifier number only and evaluated in a computer generated randomized order to avoid assessing the histology of the same patient consecutively. The whole mucosa of each section was assessed for the presence of ICSA. Positive ICSA was defined as discrete and round dark brown staining within cells in the epithelium (Figure 1a) and/or subepithelium (Figure 1b). A specimen was only categorised ICSA positive when it was scored positive by both assessors.

Inflammatory cell load and basement membrane (BM) thickness For each H\&E stained section, areas immediately below the epithelium were used for inflammatory cell counting. A total of 10 areas $\left(0.035 \mathrm{~mm}^{2}\right.$ each) were randomly selected at $5 \mathrm{X}$ magnification per slide. Areas containing submucous glands and major vessels were avoided ${ }^{(11)}$. Each area was then examined under 40X magnification for eosinophil, lymphocyte and neutrophil counting using ImageJ software (National Institute of Health, USA). The investigator (AB) that assessed the H\&E slides was blinded from the ICSA status of each patient.

Basement membrane was measured at two different points on each of the area selected in $\mathrm{H} \& \mathrm{E}$ stain using a ruler tool provided in NDP.view2 software (Hamamatsu, Japan).

\section{Statistical analysis}

Statistical analysis was performed using Prism 6 software (GraphPad Software Inc, La Jolla, CA, USA). One-way ANOVA was used to compare between the ICSA subgroups and a paired parametric t-test was performed to compare the difference between the 1st and 2nd operations within each of the subgroups. Statistical significance was defined as $p$-value $<0.05$.

\section{Results}

Patients' characteristics

A total of 34 patients (68 operations) were included in the study. Details of the demographical information is summarised in 
Table 1. Patient demographics and pre-operative characteristics.

\begin{tabular}{lc}
\hline Total number of patients & 34 \\
\hline Total number of operations & 68 \\
\hline Age & $46.1(27-70)$ \\
\hline Male: Female & $22: 12$ \\
\hline $\begin{array}{l}\text { Asthma } \\
\text { Aspirin sensitivity }\end{array}$ & $30(67.6 \%)$ \\
\hline $\begin{array}{l}\text { Smoking } \\
\text { Number of primary operations included in the study }\end{array}$ & $1(2.9 \%)$ \\
\hline $\begin{array}{l}\text { Mean number of operations prior to the study in } \\
\text { revision patients (range) }\end{array}$ & $3.1(1-10)$ \\
\hline \begin{tabular}{l} 
Mean time between operations in months (range) \\
\hline
\end{tabular} & $35.0(6-84)$ \\
\hline
\end{tabular}

Table 1. The majority of patients in this cohort were male (65\%). Asthma was common in this recalcitrant group (67.6\%) and $26.5 \%$ of patients suffered aspirin sensitivity. The mean number of previous surgical procedures of patients included in this study was 3.1 (1-10), with the mean duration between operations 35 (6-84) months.

\section{ICSA prevalence and persistence}

Within the 68 specimens assessed, 43 (63.2\%) from 26 patients (76\%) were found to be positive with ICSA (ICSA $\left.{ }^{+}\right) .18$ of these patients (52.9\%) had ICSA in specimens taken at both operations and this group was designated as ICSA ${ }^{+/+} .8$ patients (23.5\%) were ICSA negative at both operations $\left(\mathrm{ICSA}^{-/-}\right)$. There were 8 patients (23.5\%) who were only ICSA positive in one of the two operations during the study period (ICSA $\triangle$ ). Out of these 8 patients, 5 patients were only positive at the first operations $\left(\mathrm{ICSA}^{+-}\right)$and 3 were only positive in the second operations (ICSA ${ }^{1+}$ ). Among the 23 patients who were ICSA ${ }^{+}$at the first operation, $78.2 \%$ of them remained ICSA+ despite medical and surgical therapies. The mean duration between the operations in ICSA ${ }^{+/+}$ group was 33.9 (12.0-75.0) months. The demographic characteristics between the ICSA subgroups were summarised in Table 2 . There were no differences found between the use of antibiotics and corticosteroid in pre- and post-operative management between the subgroups (Table 2).

\section{Inflammatory cell load}

There was no statistical difference found in numbers of eosinophils, lymphocytes and neutrophils between the 3 ICSA subgroups. When comparing between the 2 operations within the same patients in each subgroup, the number of eosinophils was reduced from the first to the second operation in both ICSA ${ }^{-1-}$ and ICSAS $\Delta$ groups but this did not reach statistical significance. The total number of eosinophils remained the same at both time points in the ICSA ${ }^{+/+}$group (Figure 2).

\section{BM thickness}

There was no statistical difference found in BM thickness between the 3 ICSA subgroups and no significant temporal change in BM thickness noted in any subgroup.

\section{Both clinical and histological data were compared between all} 4 ICSA subgroups initially, however there were no differences
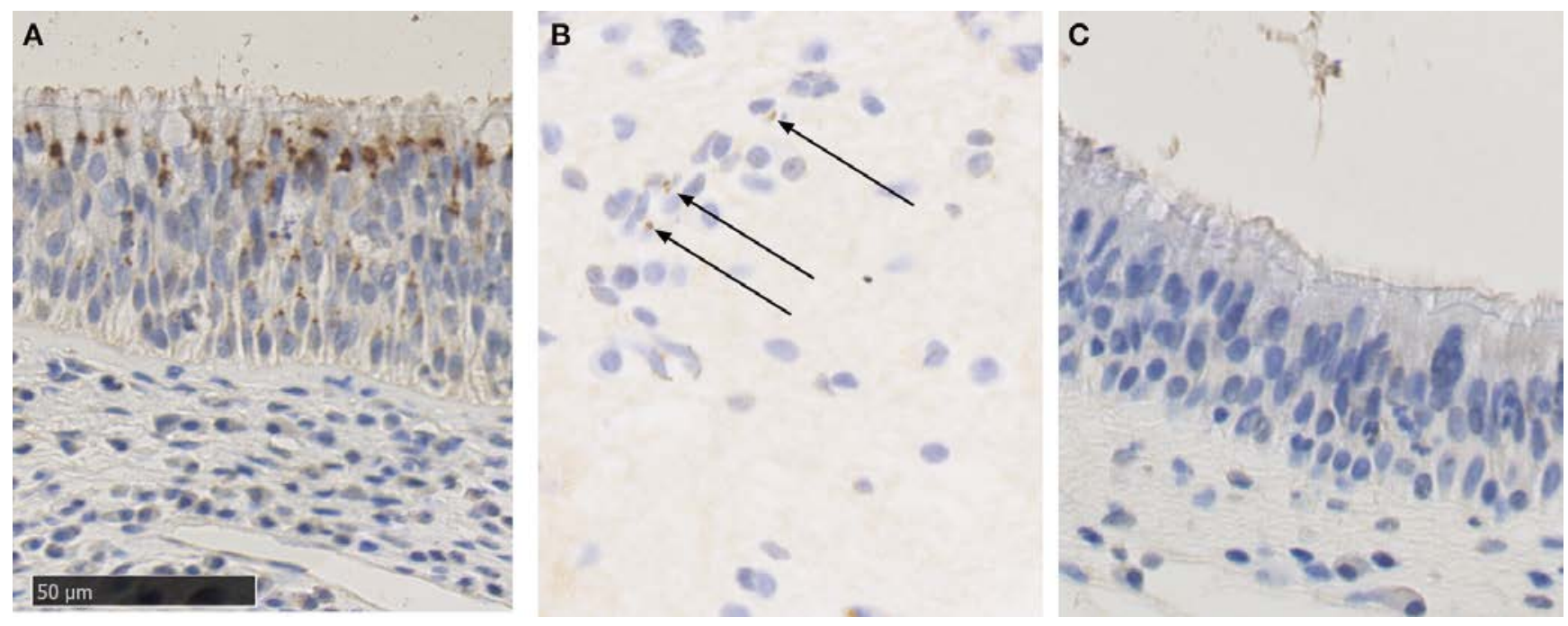

Figure 1. Immunohistochemistry stain of intracellular S. aureus (ICSA). A. An image of an ICSA+ specimen at 40X magnification. ICSA is most abundant within epithelium in the sinonasal mucosa. Positive ICSA stain is dark brown, discrete and round shaped. B. Positive ICSA staining within inflammatory cells in subepithelium digitally zoomed into 80X magnification. C. An image of an ICSA specimen at 40X magnification. 
Table 2. Demographics and clinical characteristics of each intracellular S. aureus (ICSA) subgroup.

\begin{tabular}{|c|c|c|c|c|}
\hline & $\mathrm{ICSA}^{+/+}$ & $\mathrm{ICSA}^{-/-}$ & ICSA $\triangle$ & p-value \\
\hline $\mathrm{N}$ & $18(52.9 \%)$ & $8(23.5 \%)$ & $8(23.5 \%)$ & - \\
\hline Age (range) & $45.8(29-63)$ & $46.9(27-70)$ & $46.1(33-54)$ & 0.9 \\
\hline Male: Female & $14: 4$ & $4: 4$ & $4: 4$ & 0.06 \\
\hline Asthma & $11(61.1 \%)$ & $5(62.5 \%)$ & $7(87.5 \%)$ & 0.2 \\
\hline Aspirin sensitivity & $5(27.7 \%)$ & $1(12.5 \%)$ & $3(37.5 \%)$ & 0.3 \\
\hline Smoking & $1(5.5 \%)$ & 0 & 0 & 0.4 \\
\hline Number of primary operations included in the study & $5(28 \%)$ & 0 & $3(37.5 \%)$ & 0.2 \\
\hline Mean number of operations prior to the study in revision patients (range) & $2.2(1-4)$ & $3.6(2-8)$ & $4.6(1-10)$ & 0.1 \\
\hline Mean time between operations in months (range) & $33.9(12-75)$ & $28.8(11-59)$ & $42.8(6-84)$ & 0.7 \\
\hline \multicolumn{5}{|l|}{ Pre-operative management (within 6 months) } \\
\hline Number of operations with complete pre-operative data & $26 / 36(72 \%)$ & $12 / 16(75 \%)$ & $14 / 16(88 \%)$ & - \\
\hline Topical antibiotics ${ }^{\dagger}$ & $2 / 26(8 \%)$ & $1 / 12(7 \%)$ & $0 / 14(0 \%)$ & 0.6 \\
\hline Oral antibiotics ${ }^{t, \ddagger}$ & $3 / 26(12 \%)$ & $0 / 12(0 \%)$ & $0 / 14(0 \%)$ & 0.2 \\
\hline Oral corticosteroid ${ }^{t, \S}$ & $9 / 26(35 \%)$ & $1 / 12(8 \%)$ & $2 / 14(14 \%)$ & 0.1 \\
\hline \multicolumn{5}{|l|}{ Post-operative management (18 months) } \\
\hline Number of operations with complete follow up data & $29 / 36(81 \%)$ & $13 / 16(81 \%)$ & $13 / 16(81 \%)$ & - \\
\hline Topical antibiotics $^{\dagger}$ & $12 / 29(41 \%)$ & $3 / 13(23 \%)$ & $2 / 13(15 \%)$ & 0.2 \\
\hline Short term oral antibiotics $\urcorner \|$ & 1.8 & 2.5 & 1.8 & 0.5 \\
\hline Long term (>2 weeks) oral antibiotics ${ }^{\dagger}$ & $11 / 29(38 \%)$ & $5 / 13(38 \%)$ & $5 / 13(38 \%)$ & 0.9 \\
\hline Oral corticosteroid\|l|, & 2 & 1.8 & 1.5 & 0.6 \\
\hline
\end{tabular}

${ }^{+}$A maximum of one treatment course prescribed per operation. ${ }^{\ddagger}$ Only 1 patient (in ICSA ${ }^{+/+}$group) in the whole cohort received oral antibiotics within a month prior to the operation. ${ }^{\S}$ Each corticosteroid course was defined as a 3-weeks tapering regime. " Mean number of treatment courses per operation. Note. Topical corticosteroid was prescribed to all patients post-operatively.

found between the 4 groups. Given the low number of patients in the ICSA ${ }^{+/}$and ICSA ${ }^{-/+}$groups, these data were combined and presented as one group $\left(\right.$ ICSA $\left.^{\Delta}\right)$.

\section{Discussion}

This study was conducted to evaluate the prevalence of ICSA in patients with recalcitrant CRSwNP as well as the changes in ICSA status over time. In this cohort, $76 \%$ of patients were found to have ICSA at some point during the study period. This is consistent with previous studies that report a prevalence of ICSA in patients with CRSwNP ranging between $33-89 \%^{(3-5,12,13)}$. This study also showed that in the majority of patients requiring revision surgery, ICSA persisted despite standard post-operative antibiotic treatment. In spite of ICSA persistence, there was no associated increase in eosinophils, lymphocytes, neutrophils and BM thickness found.

Differences in the prevalence rates detected by the different studies may represent differences in the disease process itself but also methodological differences. In our patient cohort, we used whole image analysis technology that reduces random sampling errors. Furthermore, the type and site of tissue harvested could differ between studies. Whereas other studies did not specify the identity of the tissue sampled, this study only examined polyp tissue. A number of different methods currently exist for the detection of ICSA. Early studies relied on Gram staining ${ }^{(4,14,15)}$ which is non-specific for $S$. aureus. More recently both Fluorescent in situ Hybridization (FISH) ${ }^{(4,12)}$ and S. aureus immunohistochemistry have been used with similar rates of sensitivity and specificity ${ }^{(16)}$. This study elected to use immunohistochemistry as it does not require fresh tissue and can be used on paraffin embedded tissue sections. It not only allowed evaluation of ICSA in both the intra- and sub-epithelium location but also permitted assessment of its association with subepithelial inflammatory cells. Disease specific and geographical differences may also explain differences in the prevalence 
A. ICSA ${ }^{4 /+}$

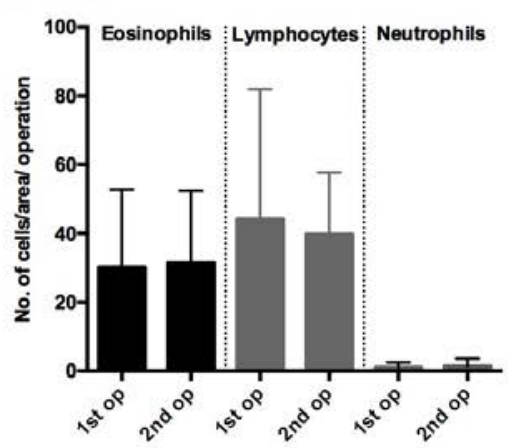

B. ICSA ${ }^{\text {t- }}$

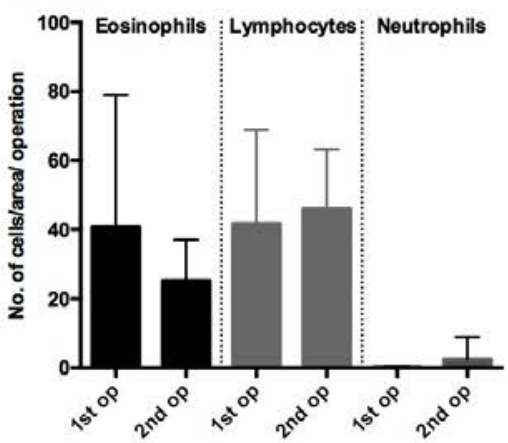

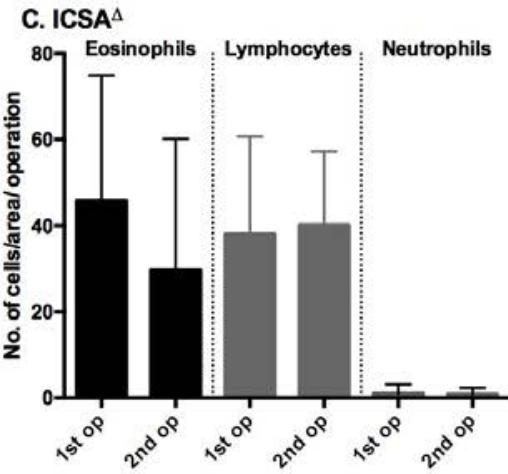

Figure 2. Comparison of the numbers of eosinophils, lymphocytes and neutrophils between 1 st and 2nd operations within intracellular S. aureus (ICSA) subgroups.

rates of ICSA seen among studies. ICSA has been repetitively found to be more prevalent in patients that have undergone revision surgeries ${ }^{(5,9)}$ and may explain the high prevalence in this study. Similarly, just as S. aureus nasal carriage has been shown to vary between different countries ${ }^{(17-21)}$ so may the prevalence of sinonasal ICSA.

When comparing the presence of ICSA between the sinonasal biopsies taken at different time points within the same patient, we found that the majority of patients who were positive for ICSA in the earlier operation remained ICSA positive at the subsequent procedure. Currently, ICSA is not routinely screened clinically and current management of CRS does not target ICSA directly. The study by Clement et al. ${ }^{(1)}$ showed that ICSA was able to persist for prolonged periods of time despite repeated systemic antibiotic treatments. Their findings together with our results again showed that current standard antibiotics regimes are ineffective for ICSA eradication. This is likely due to poor intracellular activity and penetration of currently used antibiotics ${ }^{(7)}$. Moreover, it is known that $S$. aureus alters its phenotype and growth rate once inside the host cell, further compromising the antimicrobial effect of antibiotics that are largely dependent on the microbial metabolic activity and proliferation rate $(6,22,23)$. From the data available in this study it was not possible to draw definitive conclusions on whether ICSA was present continuously or intermittently in these patients. It is possible that some of the ICSA ${ }^{+/+}$patients had eliminated ICSA at some point, however ICSA re-infection occurred subsequently due to a combination of host and pathogen factors. It is also possible that immediately post ESS the bacterial load was reduced by removing diseased sinonasal mucosa and polyps but not sufficient enough to allow complete eradication ${ }^{(8,24)}$. Despite the high rate of ICSA persistence found in our cohort, some patients were able to completely eradicate ICSA. Our study did not identify any demographic or clinical characteristics particular to this small cohort, although a larger more powered study is needed to confirm these findings.
It is likely that the virulence of different bacterial strains as well as specific host immune responses may have both contributed to ICSA eradication and molecular studies are needed to further evaluate the immune response to ICSA.

Our study showed no differences in numbers of eosinophils, lymphocytes and neutrophils between the different patient cohorts. Whilst these results indicated that the presence of ICSA did not associated with an influx of these specific cell types, we cannot exclude that ICSA presence might induce a different immune response involving other types of immune cells. There have been a number of studies performed investigating the interaction between S. aureus and non-professional phagocytes upon internalisation ${ }^{(6,22,23,25-27)}$. A down-regulation in the $S$. aureus global virulence factor regulator, accessory gene regulator (agr), has been repeatedly demonstrated ${ }^{(23,25)}$. A study conducted by Tuchscherr et al. ${ }^{(23)}$ also showed a continuous down-regulation in both agr and alpha-haemolysin gene ( $h l a)$ for up to 7 days and 28 days post intracellular S. aureus infection in cell and animal models, respectively. Other changes in S. aureus gene expression including down-regulation in enterotoxins ${ }^{(6)}$ and metabolic genes as well as up-regulation of cell wall synthesis ${ }^{(22)}$ have also been shown. Matussek et al. (26) and Li et al. (27) examined the in vitro cellular immune response to ICSA using endothelial and epithelial cells respectively. Both of these studies showed an up-regulation of a number of proinflammatory cytokines such as G-CSF, GM-CSF, IL-8 and cox2. The duration of these studies were 8 to 18 hours only and therefore may not be reflective of chronic infections such as CRS. A longer-term animal model by Tuchscherr et al. ${ }^{(23)}$ examined cytokine expression over a 28-day period showing initial upregulation of pro-inflammatory cytokines, CCL5 and CXCL11 (peak at day 7) followed by a downregulation over the course of 28 days. Overall, these studies provided evidence that once inside the mammalian cells, ICSA is able to adapt to the intracellular environment and switches its phenotype to become less virulent. This could influence the host 
immune response such that $S$. aureus is able to remain within the host mucosal tissue.

Basement membrane thickness was used as a measure for fibrosis in this study ${ }^{(11,28)}$. Pathological BM thickening was observed in all patients in this cohort and this was consistent with other studies ${ }^{(28,29)}$. Pathological BM thickening has been shown to be associated with epithelial damage and eosinophil infiltration ${ }^{28)}$. However, there was no association found between ICSA and BM thickness in this study.

The retrospective design and small sample size are limitations of this study. A prospective study with inclusion of detailed clinical data as well as regular sampling of sinonasal biopsies to closely monitor the ICSA status would be ideal to determine whether ICSA persistence was intermittent or continuous. For logistical reasons this would be difficult and result in increased patient discomfort during sampling and possibly also increase the risk of recurrent infections in patients. While harvesting tissue during ESS is the least invasive for patients, the duration between operations is variable and can be many years as observed in this study and intermediate changes in histological parameters could be missed. Overall, this is the first longitudinal study on ICSA in patients with CRSwNP to investigate its natural history under current CRS management.

\section{Conclusion}

ICSA was demonstrated within sinonasal mucosa in patients with recalcitrant CRSwNP over prolonged period of time. The presence of ICSA was not associated with an increase in eosinophils, lymphocytes and neutrophils and BM thickening, supporting the current view that ICSA can persist without inducing an inflammatory response involving these cell types. Further studies are needed to gain insight in the interaction between ICSA and host at the cellular and molecular level.

\section{Acknowledgements}

We would like to thank Ms Debbie Dyer and Ms Deanna WallisHill (Adelaide Pathology Partners) for their tremendous help in organising H\&E and IHC staining for this study. We also would like to acknowledge Dr Jim Manavis (Hanson Institute) and Centre of Neurological Disease, Hanson Institute in providing access and assistance to the NanoZoomer Platform.

This project was funded by a Project Grant to PJ Wormald from The Garnett Passe and Rodney Williams Memorial Foundation and by a scholarship to J Ou from The Garnett Passe and Rodney Williams Memorial Foundation.

\section{Authorship contribution}

Study conception and design: JO, SV; Acquisition of data: JO, AB, $A D$; Analysis and interpretation of data: JO, AD; Drafting: JO, AD; Critical revision: SV, AJP, PJW.

\section{Conflict of interest}

None relevant to this study.

\section{References}

1. Clement S, Vaudaux P, Francois P, Schrenze J, Huggler E, Kampf S, et al. Evidence of an intracellular reservoir in the nasal mucosa of patients with recurrent Staphylococcus aureus rhinosinusitis. The Journal of infectious diseases. 2005 Sep 15;192(6):1023-8.

2. Plouin-Gaudon I, Clement S, Huggler E, Chaponnier C, Francois P, Lew D, et al. Intracellular residency is frequently associated with recurrent Staphylococcus aureus rhinosinusitis. Rhinology. 2006 Dec;44(4):249-54.

3. Tan NC, Foreman A, Jardeleza C, Douglas $\mathrm{R}$, Tran H, Wormald PJ. The multiplicity of Staphylococcus aureus in chronic rhinosinusitis: correlating surface biofilm and intracellular residence. Laryngoscope. 2012 Aug;122(8):1655-60.

4. Wood AJ, Fraser JD, Swift S, PattersonEmanuelson EA, Amirapu S, Douglas RG. Intramucosal bacterial microcolonies exist in chronic rhinosinusitis without inducing a local immune response. Am J Rhinol Allergy. 2012 Jul-Aug;26(4):265-70.

5. Tan NC, Foreman A, Jardeleza C, Douglas R, Vreugde S, Wormald PJ. Intracellular Staphylococcus aureus: the Trojan horse of recalcitrant chronic rhinosinusitis? Int Forum Allergy Rhinol. 2013 Apr;3(4):261-6.

6. Tan NC, Cooksley CM, Roscioli E, Drilling AJ, Douglas R, Wormald PJ, et al. Small-colony variants and phenotype switching of intracellular Staphylococcus aureus in chronic rhinosinusitis. Allergy. 2014 Oct;69(10):136471.

7. Barcia-Macay M, Seral C, MingeotLeclercq MP, Tulkens PM, Van Bambeke F. Pharmacodynamic evaluation of the intracellular activities of antibiotics against Staphylococcus aureus in a model of THP-1 macrophages. Antimicrob Agents Chemother. 2006 Mar;50(3):841-51.

8. Drilling A, Coombs GW, Tan $\mathrm{HL}$, Pearson JC, Boase S, Psaltis A, et al. Cousins, siblings, or copies: the genomics of recurrent Staphylococcus aureus infections in chronic rhinosinusitis. Int Forum Allergy Rhinol. 2014 Dec;4(12):953-60.

9. Ou J, Drilling A, Singhal D, Tan NC, Wallis-Hill $D$, Vreugde $S$, et al. Association of intracellular Staphylococcus aureus with prognosis in chronic rhinosinusitis. Int Forum Allergy Rhinol. 2016 Aug;6(8):792-9

10. Fokkens WJ, Lund VJ, Mullol J, Bachert C, Alobid I, Baroody F, et al. EPOS 2012
European position paper on rhinosinusitis and nasal polyps 2012. A summary for otorhinolaryngologists. Rhinology. 2012 Mar;50(1):1-12.

11. Bassiouni A, Ou J, Rajiv S, Cantero D, Vreugde S, Wormald PJ. Subepithelial inflammatory load and basement membrane thickening in refractory chronic rhinosinusitis with nasal polyposis: a histopathological study. Int Forum Allergy Rhinol. 2016 Mar;6(3):248-55.

12. Corriveau MN, Zhang N, Holtappels G, Van Roy N, Bachert C. Detection of Staphylococcus aureus in nasal tissue with peptide nucleic acid-fluorescence in situ hybridization. Am J Rhinol Allergy. 2009 Sep-Oct;23(5):461-5.

13. Kim R, Freeman J, Waldvogel-Thurlow S, Roberts S, Douglas R. The characteristics of intramucosal bacteria in chronic rhinosinusitis: a prospective cross-sectional analysis. Int Forum Allergy Rhinol. 2013 May;3(5):349-54

14. Wood AJ, Fraser J, Amirapu S, Douglas RG. Bacterial microcolonies exist within the sphenoid bone in chronic rhinosinusitis and healthy controls. Int Forum Allergy Rhinol. 2012 Mar-Apr;2(2):116-21. 
15. Kim RJ, Yin T, Chen CJ, Mansell CJ, Wood A, Dunbar PR, et al. The interaction between bacteria and mucosal immunity in chronic rhinosinusitis: a prospective cross-sectional analysis. Am J Rhinol Allergy. 2013 NovDec;27(6):e183-9.

16. Tan NC, Tran HB, Foreman A, Jardeleza C, Vreugde $S$, Wormald PJ. Identifying intracellular Staphylococcus aureus in chronic rhinosinusitis: a direct comparison of techniques. Am J Rhinol Allergy. 2012 NovDec;26(6):444-9.

17. Anwar MS, Jaffery G, Rehman Bhatti KU, Tayyib M, Bokhari SR. Staphylococcus aureus and MRSA nasal carriage in general population. J Coll Physicians Surg Pak. 2004 Nov; 14(11):661-4.

18. Choi CS, Yin CS, Bakar AA, Sakewi Z, Naing NN, Jamal F, et al. Nasal carriage of Staphylococcus aureus among healthy adults. J Microbiol Immunol Infect. 2006 Dec;39(6):458-64.

19. Akmatov MK, Mehraj J, Gatzemeier A, Strompl J, Witte W, Krause G, et al. Serial home-based self-collection of anterior nasal swabs to detect Staphylococcus aureus carriage in a randomized population-based study in Germany. Int J Infect Dis. 2014 Aug;25:4-10.

20. Chen B, Dai X, He B, Pan K, Li H, Liu X, et al. Differences in Staphylococcus aureus nasal carriage and molecular characteristics among community residents and healthcare workers at Sun Yat-Sen University, Guangzhou, Southern China. BMC Infect Dis. 2015 Jul 30;15:303.

21. Abou Shady HM, Bakr AE, Hashad ME,
Alzohairy MA. Staphylococcus aureus nasal carriage among outpatients attending primary health care centers: a comparative study of two cities in Saudi Arabia and Egypt. Braz J Infect Dis. 2015 JanFeb;19(1):68-76.

22. Garzoni C, Francois P, Huyghe A, Couzinet S, Tapparel C, Charbonnier Y, et al. A global view of Staphylococcus aureus whole genome expression upon internalization in human epithelial cells. BMC Genomics. 2007 Jun 14:8:171.

23. Tuchscherr L, Medina E, Hussain M, Volker W, Heitmann V, Niemann S, et al. Staphylococcus aureus phenotype switching: an effective bacterial strategy to escape host immune response and establish a chronic infection. EMBO Mol Med. 2011 Mar;3(3):129-41.

24. Jervis-Bardy J, Boase S, Psaltis A, Foreman A, Wormald PJ. A randomized trial of mupirocin sinonasal rinses versus saline in surgically recalcitrant staphylococcal chronic rhinosinusitis. Laryngoscope. 2012 Oct;122(10):2148-53.

25. Kahl BC, Belling G, Becker P, Chatterjee I, Wardecki K, Hilgert K, et al. Thymidinedependent Staphylococcus aureus smallcolony variants are associated with extensive alterations in regulator and virulence gene expression profiles. Infect Immun. 2005 Jul;73(7):4119-26.

26. Matussek A, Strindhall J, Stark L, Rohde $M$, Geffers R, Buer J, et al. Infection of human endothelial cells with Staphylococcus aureus induces transcription of genes encoding an innate immunity response.
Scand J Immunol. 2005 Jun;61 (6):536-44.

27. Li X, Fusco WG, Seo KS, Bayles KW, Mosley EE, McGuire MA, et al. Epithelial Cell Gene Expression Induced by Intracellular Staphylococcus aureus. Int J Microbiol. 2009;2009:753278.

28. Saitoh T, Kusunoki T, Yao T, Kawano K, Kojima Y, Miyahara K, et al. Relationship between epithelial damage or basement membrane thickness and eosinophilic infiltration in nasal polyps with chronic rhinosinusitis. Rhinology. 2009 Sep;47(3):275-9.

29. Ponikau JU, Sherris DA, Kephart GM, Kern EB, Gaffey TA, Tarara JE, et al. Features of airway remodeling and eosinophilic inflammation in chronic rhinosinusitis: is the histopathology similar to asthma? J Allergy Clin Immunol. 2003 Nov;1 12(5):877-82.

\section{Peter-John Wormald \\ Department of Surgery \\ Otorhinolaryngology \\ Head and Neck Surgery \\ The University of Adelaide \\ South Australia \\ Australia}

E-mail:

peterj.wormald@adelaide.edu.au 